\title{
Protein Self-Assembly and Lipid Binding in the Folding of the Potassium Channel $\mathrm{KcsA}^{\dagger}$
}

\author{
Francisco N. Barrera, ${ }^{\ddagger}$ M. Lourdes Renart, ${ }^{\ddagger}$ José A. Poveda, ${ }^{\ddagger}$ Ben de Kruijff, ${ }^{\S}$ J. Antoinette Killian,${ }^{\S}$ and \\ José M. González-Ros*,\$ \\ Instituto de Biología Molecular y Celular, Universidad Miguel Hernández, Elche, 03202 Alicante, Spain, and Biochemistry of \\ Membranes, Bijvoetcenter, and Institute of Biomembranes, Utrecht University, Padualaan 8, $3584 \mathrm{CH}$ Utrecht, The Netherlands
}

Received April 25, 2007; Revised Manuscript Received December 13, 2007

\begin{abstract}
Moderate concentrations of the alcohol 2,2,2-trifluoroethanol (TFE) cause the coupled unfolding and dissociation into subunits of the homotetrameric potassium channel KcsA, in a process that is partially irreversible when the protein is solubilized in plain dodecyl $\beta$-D-maltoside (DDM) micelles [Barrera et al. (2005) Biochemistry 44, 14344-52]. Here we report that the transition from the folded tetramer to the unfolded monomer becomes completely reversible when KcsA is solubilized in mixed micelles composed of the detergent DDM and the lipids DOPE (1,2-dioleoyl-sn-glycero-3-phosphoethanolamine) and DOPG (1,2-dioleoyl-sn-glycero-3-[phospho-rac-(1-glycerol)]). This result suggests that lipids may act as effectors in the tetramerization of KcsA. The observed reversibility allowed the determination of the standard free energy of the folding reaction of KcsA: $\Delta G=30.5 \pm 3.1 \mathrm{kcal} \cdot \mathrm{mol}^{-1}$. We also observed that, prior to the unfolding of the tetramer, the presence of lower TFE concentrations causes the disassembly of supramolecular clusters of KcsA into the individual tetrameric molecules. Within the limits of experimental resolution, this is also a reversible process, but unlike the tetramer to monomer transition from above, the level of clustering is not influenced by the presence of solubilized lipids. These observations suggest a distinct role of the lipids in the different in vitro assembly steps (folding/tetramerization and clustering) of KcsA.
\end{abstract}

The study of the folding and stability of integral membrane proteins is the focus of an increasing interest. However, despite recent progress in the field $(1,2)$, little is still known about the molecular determinants that drive a nascent hydrophobic polypeptide synthesized in the ribosome to insert into its target lipid membrane and acquire a folded conformation and, in some cases, to associate with other biomolecules and form supramolecular complexes. As in the case of soluble proteins, relatively simple model proteins are being used to determine the general principles that govern the folding of membrane proteins $(3,4)$. One such protein, the Streptomyces lividans channel KcsA (5), belongs to the potassium channel superfamily and can be easily expressed in Escherichia coli, purified, and functionally reconstituted into lipid membranes. The crystal structure of KcsA (6) shows that the protein is arranged as a homotetramer with subunits of 160 amino acids, each comprising $\mathrm{N}$ - and C-terminal cytoplasmic domains and two transmembrane $\alpha$-helices connected by a P-loop. It has been recently assessed, both in vitro (7) and in vivo (8), that the membranebound KcsA tetramer further assembles into clusters, sug-

$\dagger$ This work was supported by grants from the Spanish DGI BFU2005-00749, BANCAJA-UMH IP/UR/01, and Generalitat Valenciana GV07/017. F.N.B. was partly supported by an EMBO short-term fellowship. M.L.R. was partly supported by a predoctoral fellowship from the Ministerio de Educación y Ciencia of Spain.

* To whom correspondence should be addressed: tel +34 966658757 ; fax +34 966658758; e-mail gonzalez.ros@umh.es.

Universidad Miguel Hernández.

$\S$ Utrecht University. gesting that in the biologically relevant state, KcsA channels may self-interact tightly. Such interaction becomes much weaker in detergent solution and indeed, only $\sim 10 \%$ of the channels remain clustered upon purification as a $\mathrm{DDM}^{1}$ solubilized protein (7).

It has been reported that the KcsA tetramer withstands high concentrations of the commonly used denaturing agents SDS (1 M), urea (7 M), guanidine hydrochloride (8 M), and guanidine isothiocyanate $(5 \mathrm{M})$ but that it becomes dissociated in the presence of a mixture of $49.5 \%$ 2,2,2-trifluoroethanol (TFE) and $0.5 \%$ trifluoroacetic acid (9). A subsequent study established that TFE alone is able to unfold KcsA (10). We have recently characterized the TFE-induced unfolding of KcsA solubilized in the detergent dodecyl $\beta$-D-maltoside (DDM) (11). Our conclusion was that the effects on the protein structure of increasing alcohol concentrations include two successive, protein-concentration-dependent cooperative transitions. The first of these transitions, occurring with $[\mathrm{TFE}]_{1 / 2}$ in the range of $23-32 \%(\mathrm{v} / \mathrm{v})$ (for the different KcsA concentrations employed), leads to the dissociation of KcsA into subunits, associated with a significant loss of $\alpha$-helical

\footnotetext{
${ }^{1}$ Abbreviations: DDM, dodecyl $\beta$-D-maltoside; DOPC, 1,2-dioleoyl$s n$-glycero-3-phosphocholine; DOPE, 1,2-dioleoyl-sn-glycero-3-phosphoethanolamine; DOPG, 1,2-dioleoyl-sn-glycero-3-[phospho-rac-(1glycerol)]; ESI-MS, electrospray ionization mass spectrometry; $\Delta G$, denaturation standard free energy in the absence of denaturant; PE, phosphatidylethanolamine; PG, phosphatidylglycerol; SDS-PAGE, polyacrylamide gel electrophoresis in the presence of sodium dodecyl sulfate; TLC, thin-layer chromatography; TFE, 2,2,2-trifluoroethanol; $[\mathrm{TFE}]_{1 / 2}$, TFE concentration at the transition midpoint.
} 
structure. This is a partly reversible process, as simple TFE dilution in these samples leads to refolding and tetramerization of the protein with an approximately $\sim 80 \%$ yield and to the recovery of the ion channel activity (11). On the other hand, the second transition, with $[\mathrm{TFE}]_{1 / 2}$ in the range of $35-45 \%$, involves a large increase in helicity and produces the irreversible TFE-induced denaturation of the protein. However, the above results were obtained for KcsA solubilized in simple detergent micelles, a milieu that only partially mimics the environment of a cell membrane, where membrane proteins are in close contact with different types of lipids and can form supramolecular complexes. Here, we study the relevance of both binding of lipids and cluster assembly in the KcsA folding process, to get insights into the role of these lipid-protein and protein-protein interactions in the folding and stability of membrane proteins. Indeed, these studies indicated that the unfolding of the KcsA tetramer to yield partly unfolded monomeric subunits was completely reversible when the DDM micelles that solubilized KcsA contained small amounts of the lipids DOPE and DOPG. Such complete reversibility allowed the calculation of the free energy of unfolding, a parameter that has been rarely obtained for oligomeric membrane proteins. We also observed that the KcsA clusters present in detergent solution can be reversibly disrupted at low TFE concentrations, in a process that is independent of the above unfolding/dissociation into subunits of KcsA.

\section{MATERIALS AND METHODS}

Protein Expression and Purification. Expression of the wild-type KcsA protein with an added N-terminal His ${ }_{6}$ tag in Escherichia coli M15 (pRep4) cells, and its purification by affinity chromatography on a $\mathrm{Ni}^{2+}-\mathrm{NTA}$ agarose column, were carried out as reported previously (12), except that a higher DDM concentration was present in the final buffer (5 mM DDM and $20 \mathrm{mM}$ HEPES, pH 7, containing 100 $\mathrm{mM} \mathrm{KCl}$ ). In addition, a gel-filtration step on a HiTrap desalting chromatography column was employed to eliminate the imidazole used to elute the protein from the $\mathrm{Ni}^{2+}-\mathrm{NTA}$ agarose column. The presence of lipids associated with purified KcsA was assessed by thin-layer chromatography (TLC) as in ref 9, as a band was observed comigrating with the negatively charged lipid PG. The protein concentration was determined by absorbance at $280 \mathrm{~nm}$, by use of a molar extinction coefficient of $34950 \mathrm{M}^{-1} \cdot \mathrm{cm}^{-1}$ for the $\mathrm{KcsA}$ monomer, obtained from the molar extinction coefficients of model compounds (13). Quantitative amino acid analysis of KcsA samples was performed, and the obtained protein concentration was compared with that determined by absorbance measurements. This allowed the correction of the absorbance concentration measurements, by the use of a correction factor of $\times 0.78$. Corrected concentration values, expressed in terms of KcsA monomers, are given in all cases.

$S D S-P A G E$. To study the degree of reversibility of the KcsA-coupled unfolding and tetramer dissociation, aliquots of KcsA $(2.5 \mu \mathrm{M})$ solubilized in DDM, supplemented with lipids (Avanti) and at different TFE concentrations, were run in $13.5 \%$ polyacrylamide gels (14). Alternatively, in SDSPAGE experiments to assess the reversibility of the dissociation of dimers of tetrameric channels ("clusters"), aliquots of $20 \mu \mathrm{L}$ of $\mathrm{KcsA}(30 \mu \mathrm{M})$ in the presence of $37 \%$ TFE, were diluted 2-fold with buffer and partially evaporated under vacuum in a Savant Speed Vac concentrator to reduce the TFE content. After reduction of the sample volumes to $10 \mu \mathrm{L}$ with a 10 -min drying step, $10 \mu \mathrm{L}$ of water was added, and samples were run in $10 \%$ polyacrylamide gels. Alternatively, we tried to reduce the TFE concentration of these samples by extensive dialysis in DDM-containing buffer. However, substantial sample precipitation was observed. Equimolar amounts of KcsA were loaded in all the wells of the gels. Samples were mixed with electrophoresis sample buffer to yield a final concentration of $35 \mathrm{mM}$ Tris, $4 \%$ glycerol, $0.01 \%$ bromophenol blue, and $70 \mathrm{mM}$ SDS, $\mathrm{pH}$ 6.8. After staining by Coomassie Brilliant Blue, the intensity of the bands was measured by densitometry. SDS-PAGE experiments were run at room temperature, without previous sample boiling.

Fluorescence Spectroscopy: (A) Emission Fluorescence Spectra. The study of the effect of DOPE/DOPG on the fluorescence of DDM-solubilized KcsA was performed on either a SLM Aminco 8000 or a Varian Cary Eclipse spectrofluorometer. For experiments on the SLM spectrofluorometer, the scan rate was $60 \mathrm{~nm} / \mathrm{min}$ and a $0.5-\mathrm{cm}$ quartz cuvette was used. For the experiments on the Varian spectrofluorometer, the scan rate was $600 \mathrm{~nm} / \mathrm{min}$, the number of averaged scans was five, and a 1-cm quartz cuvette was employed. Very similar results were obtained with both spectrofluorometers. In all the experiments, the excitation wavelength was $280 \mathrm{~nm}$, the measured emission range was $300-400 \mathrm{~nm}$, and the temperature was $25^{\circ} \mathrm{C}$. Experiments were recorded with polarizers in a cross-oriented configuration $\left(\mathrm{Ex}_{\mathrm{pol}}=90^{\circ}, \mathrm{Em}_{\mathrm{pol}}=0^{\circ}\right)$ that provides maximal suppression of scattering artifacts (15). Buffer contribution was subtracted in all cases. The study of the TFE-induced unfolding of KcsA in mixed micelles of DDM and DOPE/DOPG (7:3) was performed with both spectrofluorometers. The transition was monitored by following the changes in the intensity-weighted average emission wavelength, $\langle\lambda\rangle(16)$ :

$$
\langle\lambda\rangle=\frac{\sum I_{i} \lambda_{i}}{\sum I_{i}}
$$

where $I_{i}$ is the fluorescence intensity measured at a wavelength $\lambda_{i}$. The use of the $\langle\lambda\rangle$ parameter (expressed in nanometers) is particularly suited to follow small spectral changes irrespective of any particular wavelength, as it integrates information from the fluorescence intensity emitted at all the wavelengths recorded in the spectrum.

(B) Thermal Unfolding. Thermal unfolding experiments of KcsA were carried out in the Varian spectrofluorometer at a temperature up-scan rate of $0.6{ }^{\circ} \mathrm{C} / \mathrm{min}$. Experiments were performed with a final KcsA concentration of $1.3 \mu \mathrm{M}$, whether solubilized in DDM or in mixed micelles of DDM and DOPE/DOPG (7:3). The excitation wavelength was 280 $\mathrm{nm}$ and the fluorescence was recorded at $340 \mathrm{~nm}$. The slit widths for both excitation and emission were $5 \mathrm{~nm}$ and the integration time was $5 \mathrm{~s}$. The study of the unfolding of tetrameric KcsA was performed with protein in the presence of $16 \%$ TFE, where KcsA remains in its native tetrameric state (11). The experiment was repeated in the presence of $35 \%$ TFE, where KcsA remains as a partly unfolded monomer. To establish the degree of refolding in the different 
conditions, additional samples of unfolded, monomeric KcsA (i.e., treated with $35 \%$ TFE) were prepared at a KcsA concentration of $2.2 \mu \mathrm{M}$, and after 1 min incubation, the samples were appropriately diluted with DDM buffer to reach both the protein concentration employed in the unfolding experiments $(\mathrm{Kcs} A, 1.3 \mu \mathrm{M})$ and a final TFE concentration of $16 \%(\mathrm{v} / \mathrm{v})$; then the thermal unfolding experiments were performed.

(C) Anisotropy Measurements. Fluorescence anisotropy was recorded in a Perkin-Elmer LS 55 spectrofluorometer. The fluorescence steady-state anisotropy, $\langle r\rangle$, is defined by (17)

$$
\langle r\rangle=\frac{I_{\mathrm{V}}-G I_{\mathrm{H}}}{I_{\mathrm{V}}+2 G I_{\mathrm{H}}}
$$

where $I_{\mathrm{V}}$ and $I_{\mathrm{H}}$ are, respectively, the parallel and perpendicular components of the polarized fluorescence emission, with excitation polarized vertically. The $G$ factor, which corrects for the transmissivity bias introduced by the detection system, had a value of 1.15 . The excitation wavelength was $295 \mathrm{~nm}$ and the emission wavelength was $340 \mathrm{~nm}$. The slits widths were $15 \mathrm{~nm}$ for both the excitation and emission monochromators, and the acquisition average time was $3 \mathrm{~s}$. The temperature was $25^{\circ} \mathrm{C}$. Each reported anisotropy value is the average of 5-8 independent measurements.

(D) Free Energy Determination. The sigmoidal transitions monitoring the TFE-induced conversion from tetramers to monomers were fitted to

$$
X=\left(X_{\mathrm{N}}+X_{\mathrm{D}} \mathrm{e}^{(-\Delta G / R T)}\right) /\left(1+\mathrm{e}^{(-\Delta G / R T)}\right)
$$

where $R$ is the gas constant, $T$ is the temperature in kelvins, and $X_{\mathrm{N}}\left(X_{\mathrm{N}}=\alpha_{\mathrm{N}}+\beta_{\mathrm{N}}[\mathrm{TFE}]\right)$ and $X_{\mathrm{D}}\left(X_{\mathrm{D}}=\alpha_{\mathrm{D}}+\beta_{\mathrm{D}}[\mathrm{TFE}]\right)$ are the corresponding fractions of the folded $(\mathrm{N})$ and unfolded (D) states, respectively, for which a linear relationship with denaturant is assumed, $\beta_{\mathrm{N}}$ and $\beta_{\mathrm{D}}$ being the slopes of the folded and unfolded plateaus, respectively. According to the linear extrapolation model (18), $\Delta G=m\left([\mathrm{TFE}]_{1 / 2}-\right.$ [TFE] $)-R T \ln \left(\left(n^{n} / 2^{n-1}\right) C_{\mathrm{t}}^{n-1}\right)$, where $\Delta G$ is the standard free energy of denaturation in the absence of denaturant, $m$ is the slope of the curve (19), [TFE] is the denaturant concentration, $[\mathrm{TFE}]_{1 / 2}$ is the one at the midpoint of the transition, $n$ is the oligomeric state of the folded species, and $C_{\mathrm{t}}$ is the concentration of protein expressed in monomer equivalents.

Solubilization Measurements. The turbidity reduction caused by DDM solubilization of the liposomes, was monitored by absorbance measurements at $400 \mathrm{~nm}$. The measurements were carried out in a Camspec M350 double beam UV-visible spectrophotomer with 1-cm quartz cuvettes. Experiments were carried out at $25{ }^{\circ} \mathrm{C}$.

Analytical Ultracentrifugation. Sedimentation velocity experiments were conducted in a Beckman Optima XL-I ultracentrifuge (Beckman Coulter) with an An50Ti eighthole rotor and double-sector Epon-charcoal centerpieces. KcsA samples were centrifuged at $40000 \mathrm{rpm}$, at $20^{\circ} \mathrm{C}$, and the absorbance at $280 \mathrm{~nm}$ was followed. Differential sedimentation coefficient distributions, $c(s)$, were calculated by least-squares boundary modeling of sedimentation velocity data by using the program SEDFIT $(20,21)$. This analysis yielded sedimentation coefficients, $s$, for the particular conditions of each experiment. To compare the $s$ values obtained at different TFE concentrations, it was necessary to correct for the influence of the TFE on the viscosity $(\eta)$ and density $(\rho)$ of the solutions (22). Such corrections were carried out using the program SEDNTERP (http://www.rasmb.bbri.org/) and the results were given as the sedimentation coefficients in standard conditions, $S_{20, w}$. The apparent mass of the bands was calculated from the $S_{20, w}$ value, assuming a spherical shape for the particles. The KcsA concentration employed in samples of DDM-solubilized KcsA was $4 \mu \mathrm{M}$. In samples of KcsA in mixed micelles, higher protein concentrations were required to obtain a good signal-to-noise ratio. Hence, the KcsA concentration used was $6 \mu \mathrm{M}$, except in the case of samples at $0 \%$ TFE, which needed a KcsA concentration of $12 \mu \mathrm{M}$ to reach a similar signal-to-noise ratio.

\section{RESULTS}

Initial Exploration of the Effect of Lipids on the Refolding of KcsA. KcsA has been shown to be remarkably stable in SDS and to migrate as a homotetramer in SDS-PAGE gels $(9,10,12)$. Thus, SDS-PAGE provides a simple means to study the effects of destabilizing agents such as TFE on the oligomerization state of KcsA. However, this is not exempt from limitations, as the presence of SDS and its effects on KcsA stability make it difficult to compare the electrophoretic results with those obtained spectroscopically in the absence of SDS. Also, the protein concentration dependence of the effects of TFE on KcsA requires that the SDS-PAGE experiments be conducted at low concentrations used in the spectroscopic monitoring of the process. Finally, the presence of TFE in the electrophoretic samples causes the lighter sample components (monomers) to appear as faint bands in the gels or not at all (10), particularly at the low protein concentrations used in this work. However, the TFE-induced dissociation of the KcsA tetramer into monomers has been independently assessed by analytical ultracentrifugation experiments (11). Nevertheless, the TFE-induced disappearance of the KcsA tetramer can be reliably measured by densitometry, and in spite of the above limitations, we used SDS-PAGE in a prospective study to evaluate whether the presence of lipids influences the efficiency of KcsA tetramerization. Accordingly, samples of DDM-solubilized $\mathrm{Kcs} \mathrm{A}$ in the presence of a TFE concentration causing full monomerization [33\% TFE (v/v)] were diluted below unfolding-inducing TFE concentrations, with buffer supplemented with different lipids, namely, DOPC, DOPE, and DOPG and several of their binary mixtures. All lipids tested were found to improve moderately the tetramerization efficiency with respect to that seen in plain DDM buffer, leading to an increased recovery of the KcsA tetrameric band. Figure 1 illustrates the results obtained for a 7:3 molar mixture of DOPE and DOPG, which in our hands was the most efficient lipid mixture tested. Moreover, the intensity of the recovered tetrameric band seems practically identical, within experimental error, to that of the native protein, thus suggesting that in DDM-solubilized samples, the TFEinduced monomerization of KcsA could be completely reversed upon removal of TFE when in the presence of DOPE and DOPG. Control experiments showed that the presence of DOPE and DOPG did not alter the Coomassie staining of the tetrameric band of KcsA (data not shown), 
A

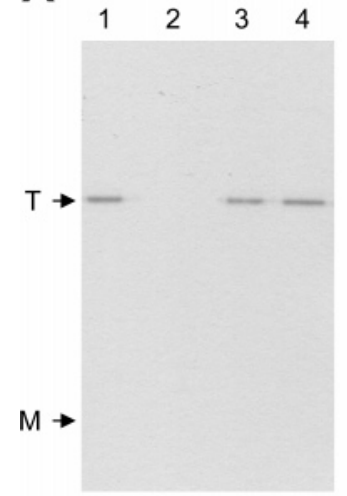

B

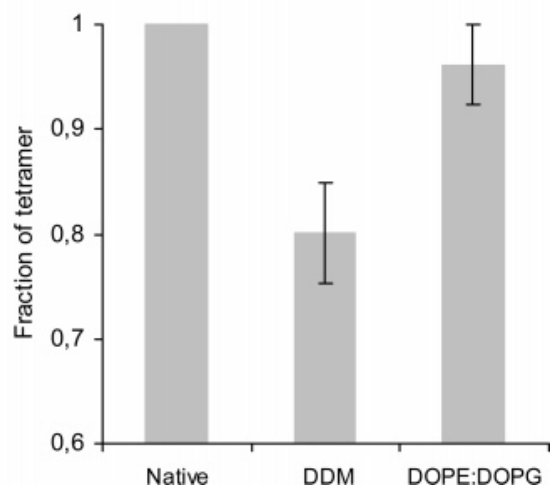

FIGURE 1: SDS-PAGE analysis of KcsA tetramerization. (A) Lane 1: Control DDM-solubilized KcsA; at the protein concentration used in these experiments $(2.5 \mu \mathrm{M})$, the characteristic KcsA tetramer (T) is the only significant band to be seen in the gels upon Coomassie staining. The intensity of this control tetrameric band is essentially identical to that in the presence of TFE concentrations up to $12 \%(\mathrm{v} / \mathrm{v})$ (not shown). $\mathrm{M}$ denotes the position in the gel where KcsA monomers would be expected to appear. Lane 2: DDM-solubilized KcsA in the presence of 33\% TFE (v/v), which causes complete dissociation into subunits of the KcsA tetramer. The band arising from the KcsA monomers is not observed under these conditions because its detection is hampered by the presence of TFE $(10,11)$. Lane 3: DDM-solubilized KcsA was first treated with 33\% TFE as in lane 2 and then diluted with DDM buffer to a final TFE concentration of $10 \%(\mathrm{v} / \mathrm{v})$ to allow reassociation of the KcsA subunits back into tetramers. Lane 4: Sample prepared as in lane 3 but in the presence of DOPE/DOPG (7:3) at a molar lipid to KcsA ratio of 1000:1 in the dilution buffer to assess lipid effects. All wells were loaded with the same amount of KcsA. (B) The intensity of the gel bands was determined by densitometry and the fraction of tetramer recovered in each sample was estimated by comparison with lane 1 . The experiment was performed four times in different gels, with different protein and lipid stocks. The normalizations were made to lane 1 of each of the gels, and the standard deviation bar is shown.

and therefore, its SDS-PAGE quantification is not affected by the presence of lipids. Interestingly, the two lipids employed were shown to favor membrane association and tetramerization of KcsA in an in vitro translation study (23).

Optimization of a Mixed Micelle System. The above results prompted us to characterize in more detail the unfolding and refolding of KcsA solubilized in a mixed micelle system composed of DDM and DOPE/DOPG (7:3). The use of mixed micelles, where both lipids and protein are completely solubilized by the detergent, allows us to overcome important spectroscopic artifacts derived from the presence of lipid vesicles. Accordingly, we decided to determine the experi- mental conditions adequate to attain mixed micelles of detergent and lipid. As we had observed that lipids can influence the refolding of KcsA, it seemed reasonable to assume that lipid molecules must be interacting with KcsA at a given stoichiometry. To determine the minimal amount of lipid required to saturate such binding to the protein, the changes in the intrinsic fluorescence of solubilized KcsA induced by the lipids DOPE/DOPG (7:3) were monitored (Figure 2A). We observed that at low lipid concentrations, the intrinsic fluorescence of KcsA increased linearly, reaching saturation at a DOPE/DOPG concentration as low as $\sim 2$ $\mu \mathrm{M}$. At all the different protein concentrations assayed $(0.2-4 \mu \mathrm{M})$, saturation of the binding curves was reached at DOPE/DOPG molar concentrations that never exceeded 15-20-fold the KcsA concentration (data not shown). Therefore, in order to work in an optimal situation of saturated lipid binding, molar lipid to protein ratios equal or greater than 15:1 were employed in all subsequent experiments.

We also performed turbidity experiments to assess whether a DDM concentration of $5 \mathrm{mM}$ could solubilize effectively the lipid vesicles at the lipid concentrations employed in Figure 2A. Accordingly, we recorded absorbance spectra of samples with a fixed DOPE/DOPG concentration of $500 \mu \mathrm{M}$ (much higher than that required to saturate the KcsA lipid binding at any of the conditions employed) in the presence of increasing concentrations of DDM. We observed that the presence of small amounts (in the micromolar range) of detergent induced an abrupt decrease in the absorbance of the samples (Figure 2B), likely caused by a reduction in the number and/or the size of the liposomes (24). At DDM concentrations near the detergent critical micelle concentration, $0.16 \mathrm{mM}$ (25), a second phase of solubilization was observed, characterized by the presence of a small shoulder, which has been reported to indicate the onset of solubilization (26). Finally, a stabilization of the absorbance was observed above 1.5-2 mM DDM, indicating the minimal detergent concentration required to completely solubilize the liposomes. This observation confirmed that at $5 \mathrm{mM}$ DDM, the usual detergent concentration in our experiments, the added DOPE/ DOPG (7:3) liposomes were fully solubilized and homogeneous mixed micelles were formed.

Unfolding of KcsA in Mixed Micelles. Changes in the protein intrinsic fluorescence were used to monitor the TFEinduced unfolding of KcsA solubilized in mixed micelles. Final concentrations for all components were $2.5 \mu \mathrm{M} \mathrm{KcsA}$, $5 \mathrm{mM}$ DDM, and $50 \mu \mathrm{M}$ DOPE/DOPG (Figure 3A). The addition of TFE up to $40 \%(\mathrm{v} / \mathrm{v})$ resulted in a sigmoidal decrease of the fluorescence intensity (data not shown) and in a small spectral red shift. In contrast to that seen for KcsA solubilized in plain DDM micelles, the red shift caused by the TFE in the mixed micelles was completely reversed upon dilution, as the normalized spectrum of a sample of KcsA at $10 \%$ TFE, fully overlapped with that of a sample unfolded with 30\% TFE and subsequently diluted with buffer to $10 \%$ TFE (Figure 3B). Additional experiments allowed us to establish that very similar results were obtained in unfolding titrations performed either with native KcsA or with unfolded KcsA (in 30\% TFE) that had been diluted to 5\% TFE (Figure 3C). These results suggest that the TFE-induced unfolding of KcsA was indeed fully reversible in mixed micelles of DOPE/DOPG (7:3) and DDM. Accordingly, the obtained 

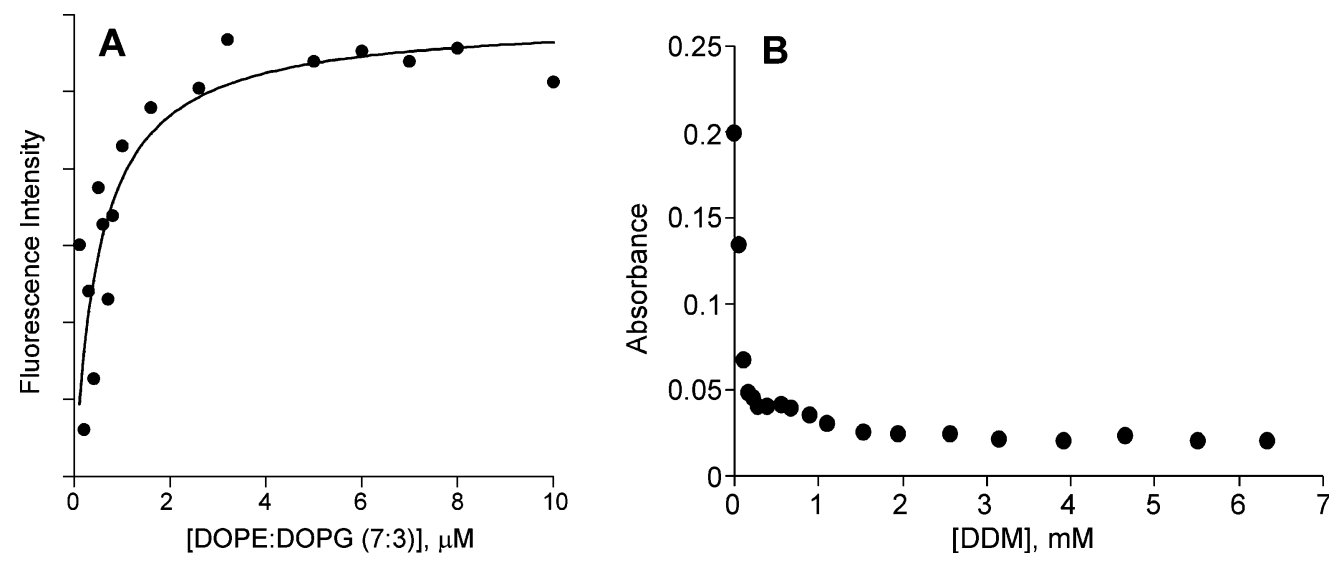

FIGURE 2: Optimization of mixed micelle conditions. (A) Fluorescence spectra of samples of KcsA ( $0.15 \mu \mathrm{M})$ solubilized in $5 \mathrm{mM}$ DDM, were recorded in the presence of increasing concentrations of DOPE/DOPG (7:3). The changes in the fluorescence intensity (arbitrary units) at $335 \mathrm{~nm}$ are shown. Excitation wavelength was $280 \mathrm{~nm}$. The line drawn is a mere guide to the eye. (B) DDM solubilization of DOPE/DOPG liposomes. The physical state of liposomes of DOPE/DOPG (7:3) was assessed at increasing concentrations of DDM following the turbidity of the samples, by measurement of the absorbance at $400 \mathrm{~nm}$. Lipid concentration was $500 \mu \mathrm{M}$. The buffer was $20 \mathrm{mM}$ HEPES, $\mathrm{pH} 7$, and $100 \mathrm{mM} \mathrm{KCl}$. All experiments were carried out at $25{ }^{\circ} \mathrm{C}$.

fluorescence unfolding transitions were fitted to an equation describing a two-state reversible transition (eq 3 ) where the native tetramer is converted to unfolded monomers (27). It should be noted that the assumption of full reversibility of the unfolding/refolding process is a strict requirement for this analysis. Then, data such as that shown in panels $\mathrm{A}$ and $\mathrm{C}$ of Figure 3 were independently fitted to eq 3 and found to yield similar values for both, native and refolded KcsA (see caption). In particular, the native KcsA samples shown in panel A yielded $[\mathrm{TFE}]_{1 / 2}=23.7 \%(\mathrm{v} / \mathrm{v})$ and $m=2.7$ $\mathrm{kcal} \cdot \mathrm{mol}^{-1} \cdot \mathrm{M}^{-1}$, while those from panel $\mathrm{C}$ were $[\mathrm{TFE}]_{1 / 2}=$ $25.3 \%(\mathrm{v} / \mathrm{v})$ and $m=2.4 \mathrm{kcal} \cdot \mathrm{mol}^{-1} \cdot \mathrm{M}^{-1}$. Finally, the estimated values for the refolded samples, included also in panel $\mathrm{C}$, were $[\mathrm{TFE}]_{1 / 2}=26.1 \%(\mathrm{v} / \mathrm{v})$ and $m=2.1$ $\mathrm{kcal} \cdot \mathrm{mol}^{-1} \cdot \mathrm{M}^{-1}$. All these results were used to calculate an averaged value for the stability of the KcsA tetramer: $\Delta G$ $=30.5 \pm 3.1 \mathrm{kcal} \cdot \mathrm{mol}^{-1}$ (see Materials and Methods). Interestingly, the $[\mathrm{TFE}]_{1 / 2}$ value obtained here for KcsA in mixed micelles was slightly lower than that determined for KcsA in plain DDM micelles under comparable conditions [28.7\% TFE (11)], suggesting that the presence of the solubilized lipids makes the tetrameric protein slightly more susceptible to TFE.

In order to further confirm the reversibility of the tetramer to monomer transition, we studied the thermal unfolding profiles from both native and refolded KcsA samples. Also, in order to account for a possible role of lipids in the unfolding/refolding equilibrium, these experiments were performed with KcsA solubilized either in DDM micelles or in the mixed micelle system. Figure 4A shows that KcsA solubilized in plain DDM micelles and in the presence of $16 \%$ TFE (v/v) (see caption to Figure 4), where the protein is in the tetrameric state (see Figure $3 \mathrm{~A}$ and ref 11 ) undergoes a sigmoidal loss of fluorescence with temperature, with a midpoint at around $70^{\circ} \mathrm{C}$. As expected, when the experiment was performed with a sample treated with $35 \%$ TFE, which causes the unfolding and the dissociation into monomers of the tetrameric KcsA, no sigmoidal transition was observed, indicating that the folded core of the protein had been lost. Nonetheless, when a concentrated sample was first treated with 35\% TFE and then diluted to lower the TFE concentration to $16 \%$ (and to a protein concentration identical to that of the two former samples), a full recovery of the sigmoidal transition was seen in the mixed micelle DOPE/DOPG (7: 3) samples (Figure 4B), which was not observed for KcsA solubilized in plain detergent micelles. These results agree with previous data that show only partial reversibility of the coupled unfolding and monomerization of KcsA when solubilized in DDM (11). Taken together with the results shown in Figure 3, these observations indicate that indeed, the presence of DOPE and DOPG (7:3) in the micelles makes the folded tetramer to unfolded monomer transition fully reversible, in agreement with the preliminary SDS-PAGE estimates in Figure 1.

$K c s A$ Clustering. It has been recently reported that KcsA forms supramolecular assemblies or clusters into which its ion channel electrophysiological properties are significantly changed $(7,8)$. For KcsA reconstituted into asolectin lipid vesicles, a high proportion of the KcsA tetramers assemble into these clusters, while in detergent solution, only a small percentage of the KcsA channels $(\sim 10 \%)$ remain as such (7). However, neither the previous KcsA unfolding studies $(9-11,28)$ nor the present fluorescence spectral measurements of KcsA solubilized in mixed micelles at different TFE concentrations (Figure 3A) have detected any distinct transition that could be ascribed to the possible TFE-induced dissociation of KcsA multimers into individual KcsA tetramers. This could be due to several reasons: (i) the process is not experimentally detectable, because either the cluster rupture is spectroscopically silent, or the percentage of KcsA channels that is forming part of these supramolecular structures is too low; (ii) the TFE disrupts the multimers in a linear, noncooperative fashion; or (iii) the cluster dissociation occurs concomitantly with the tetramer unfolding.

In order to gather more information on this subject, we monitored the TFE-induced unfolding of KcsA by means of a different spectroscopic variable, the intrinsic fluorescence anisotropy, $\langle r\rangle$, which is highly sensitive to changes in the dynamics of the indole moiety of the tryptophan residues in the protein. Again, these experiments were carried out on KcsA solubilized both in plain detergent micelles and in the mixed micelles defined above. For the DDM-solubilized KcsA (Figure 5A), we observed that the anisotropy changes monitored clearly the tetramer to monomer transition, with 

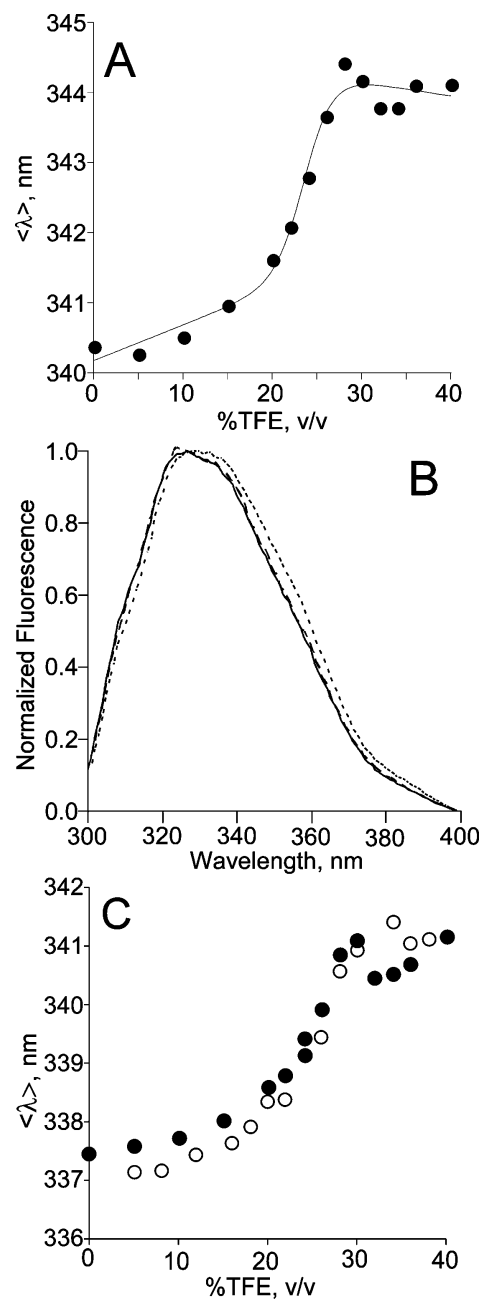

FIGURE 3: Unfolding and refolding of KcsA in mixed micelles. Samples of KcsA in mixed micelles composed of DDM $(5 \mathrm{mM})$ and DOPE/DOPG $(50 \mu \mathrm{M})$, were incubated with different TFE concentrations and the fluorescence spectra were recorded. (A) Fluorescence intensity-weighted average emission wavelength, $\langle\lambda\rangle$, at increasing TFE concentrations. The line corresponds to the fitting to eq 3 , according to ref 18 . KcsA concentration was $2.5 \mu \mathrm{M}$. (B) The emission spectra of KcsA in the presence of $10 \%$ TFE (v/v) (folded KcsA, solid line) and 30\% TFE (v/v) (unfolded KcsA, dotted line) are shown. Also, the spectrum of a KcsA sample first treated with $30 \%$ TFE and then diluted 3-fold in buffer to allow refolding is depicted (dashed line). The spectra have been normalized to the intensity maxima in order to facilitate spectral shape comparison. The spectrum of KcsA in the absence of TFE was very similar to that obtained at $10 \%$ TFE (v/v) and is not shown here for the sake of clarity. (C) TFE-unfolding titration of refolded KcsA. Samples of KcsA first treated with 30\% TFE (v/v) and then diluted with buffer to reduce the denaturant concentration to $5 \%$ $(\mathrm{v} / \mathrm{v})$ were used in these experiments. The results $(\mathrm{O})$ are compared with those obtained for a TFE titration of native KcsA samples under otherwise identical conditions $(-$ ) KcsA concentration was $0.4 \mu \mathrm{M}$. Buffer was $20 \mathrm{mM}$ HEPES and $100 \mathrm{mM} \mathrm{KCl}, \mathrm{pH} 7$. Excitation wavelength was $280 \mathrm{~nm}$ and the temperature was 25 ${ }^{\circ} \mathrm{C}$. Noncorrected spectra for the experiments in panels $\mathrm{A}$ and $\mathrm{C}$ were obtained on different spectrofluorometers (SLM Aminco 8000 and Varian Cary Eclipse, respectively), which accounts for the observed differences in the $\langle\lambda\rangle$ values. Fitting of the curve in panel A, performed with different slopes in the unfolded plateau, is provided as Supporting Information.

a $[\mathrm{TFE}]_{1 / 2}$ of $29.6 \% \pm 0.9 \% \mathrm{TFE}(\mathrm{v} / \mathrm{v})$, a value very similar to that previously determined by changes in fluorescence intensity, $\langle\lambda\rangle$, and circular dichroism (11). However, unlike those studies, we observed an additional cooperative transi-
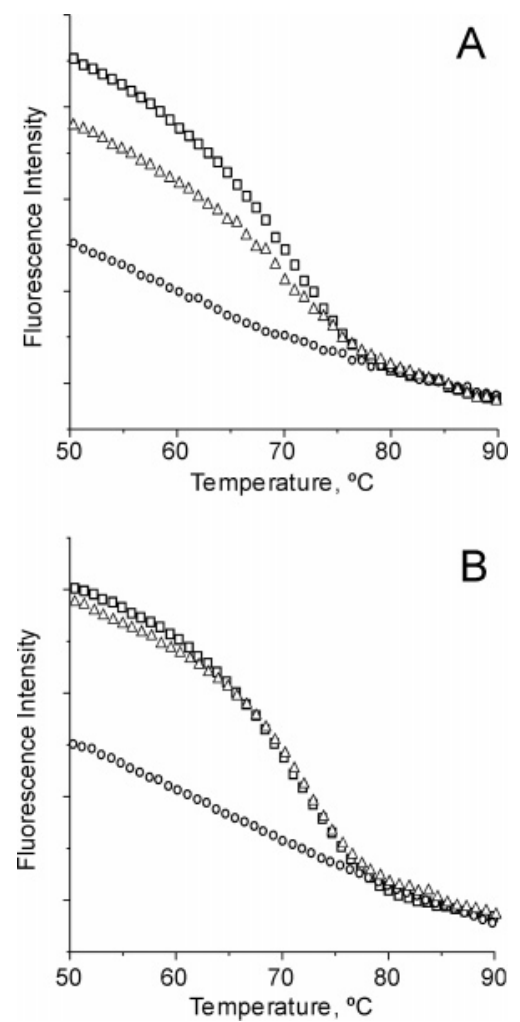

FIGURE 4: Thermal unfolding of KcsA. Reversibility of the coupled dissociation and unfolding of the tetrameric KcsA was assayed for the protein solubilized in (A) plain detergent micelles and in (B) mixed micelles of DDM and DOPE/DOPG (7:3). Thermal unfolding of the following samples was studied: tetrameric KcsA in $16 \%$ TFE ( $\square$ ), unfolded and monomeric KcsA in 35\% TFE (O), and KcsA samples that were first unfolded in 35\% TFE and subsequently diluted to $16 \%$ TFE $(\triangle)$ to assess the degree of refolding. Final KcsA concentration was $1.3 \mu \mathrm{M}$ in all cases, and the lipid concentration was $25 \mu \mathrm{M}$. The fluorescence intensity is given in arbitrary units. The use of $16 \%$ TFE is particularly suited for these experiments, as it reduces the observed thermal midpoint to more amenable values (e.g., in the absence of TFE the midpoint is near $100{ }^{\circ} \mathrm{C}$ ) without causing dissociation of the tetramer.

tion appearing at lower TFE concentrations, with an estimated $[\mathrm{TFE}]_{1 / 2}$ of $12.2 \%$ TFE $(\mathrm{v} / \mathrm{v})$. As circular dichroic results have shown that the secondary structure of KcsA remains constant in the $0-20 \%$ TFE range (11), this transition must be monitoring the conversion between two KcsA states with a very similar helical structure. Although not too relevant to the main goals of this report, the transition leading to the irreversible denaturation of the unfolded KcsA monomers, which occurs at higher TFE concentrations $\left\{[\mathrm{TFE}]_{1 / 2}\right.$ of $\sim 45 \%$ TFE (v/v) (11)\}, could also be detected by monitoring the fluorescence anisotropy, but it will not be considered any further in this paper (data not shown).

When the experiment was performed with KcsA solubilized in mixed micelles of DDM and DOPE/DOPG (7:3), an anisotropy profile was obtained where the two relevant sigmoidal transitions could also be distinguished (Figure 5B), although the plateau between the two transitions was shorter than that seen in the plain DDM micellar system. The determined $[\mathrm{TFE}]_{1 / 2}$ values $[9.0 \% \pm 0.5 \% \mathrm{TFE}(\mathrm{v} / \mathrm{v})$ for the first transition and $26.6 \% \pm 1.1 \%$ TFE (v/v) for the monomerization transition] were lower than those determined for the DDM-solubilized samples, again suggesting that the TFE resistance of KcsA is somewhat reduced by the presence of the DOPE/DOPG mixture. 

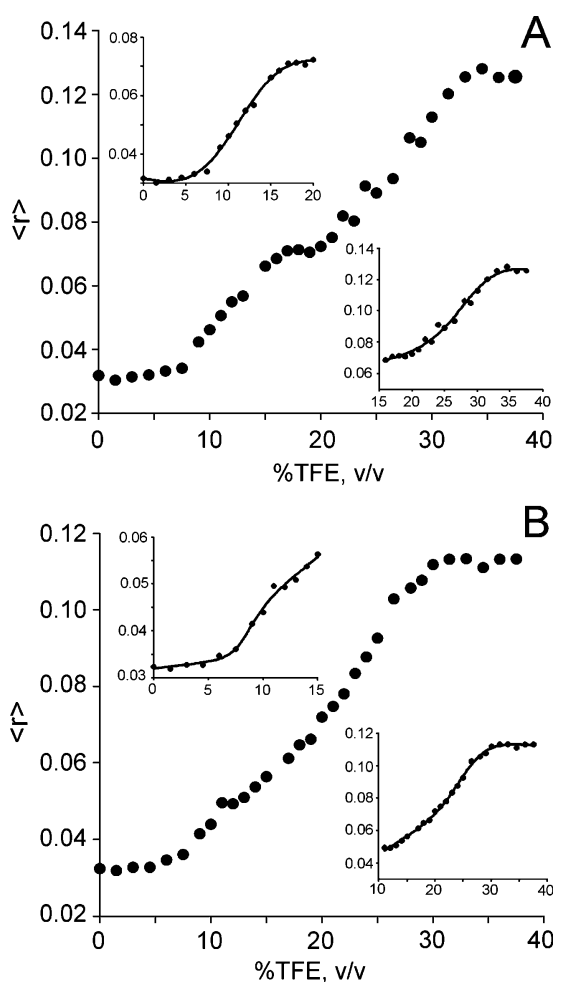

FIGURE 5: KcsA fluorescence anisotropy changes in the presence of TFE. (A) DDM-solubilized KcsA and (B) KcsA solubilized in mixed micelles of DDM and DOPE/DOPG (7:3) were employed at a protein concentration of $1.5 \mu \mathrm{M}$. The lipid to protein ratio was 15:1. Fittings of the experimental data to a two-state model (eq 3) for the cluster disassembly (upper left inset) and the tetramer dissociation (lower right inset) processes are shown in each panel. Experiments were recorded at $25^{\circ} \mathrm{C}$. Excitation wavelength was $295 \mathrm{~nm}$, and emission wavelength was $340 \mathrm{~nm}$. Buffer was 100 $\mathrm{mM} \mathrm{KCl}$ and $20 \mathrm{mM}$ HEPES, $\mathrm{pH}$ 7. The results are the average of three independent experiments.

To assess whether this new transition at low TFE concentrations was indeed monitoring the disruption of the KcsA clusters, we performed analytical ultracentrifugation experiments at different TFE concentrations. Sedimentation velocity experiments of DDM-solubilized KcsA in the absence of TFE (Figure 6A) showed a profile with a major band sedimenting at $6.6 \mathrm{~S}$. This value corresponds to an apparent molecular mass of $109.3 \mathrm{kDa}$, which is compatible with the mass of a KcsA tetramer, $76.4 \mathrm{kDa}$ (160 amino acids plus 12 additional $\mathrm{N}$-terminal amino acids of the $\mathrm{His}_{6}$ tag, per monomer), bound to a reasonable number of DDM molecules (7). This band showed a shoulder at low $S_{20 \text {,w }}$ values, likely derived from the presence of a small population of $\operatorname{Kcs} A$ monomer $(7,12)$. We also observed a second band, with a $S_{20, w}$ value of $10.5 \mathrm{~S}$, and a tiny but highly reproducible band with a $S_{20, \mathrm{w}}$ of $14.4 \mathrm{~S}$ (see inset). The calculated molecular masses, 217.8 and $349.9 \mathrm{kDa}$, respectively, suggest that these populations could correspond to the supramolecular assembly of two and three KcsA channels (i.e., KcsA clusters). When the experiment was performed in the presence of $6 \% \mathrm{TFE}(\mathrm{v} / \mathrm{v})$ (Figure 6B), we observed that the sedimentation coefficient of all the bands was lowered, likely due to the ability of the TFE to partly sequester the detergent micelles that surround the transmembrane regions of the protein, reducing then the apparent mass of the complex (7). Interestingly, at $12 \% \mathrm{TFE}(\mathrm{v} / \mathrm{v})$, the area of the bands assigned to the KcsA clusters decreased (Figure
6C), being no longer present at $18 \%$ TFE (Figure 6D). As these two TFE concentrations correspond to the midpoint and the higher plateau of the first anisotropy transition, respectively (Figure 5A), these results indicate that the anisotropy is indeed monitoring the disruption of KcsA channel clusters, which exist mainly in the form of dimers under our detergent-solubilized conditions.

These experiments were also performed with KcsA solubilized in mixed micelles of DDM and DOPE/DOPG $(7: 3)$ (Figure $6 \mathrm{E}-\mathrm{H}$ ). In the latter samples, the sedimentation profile obtained in the absence of TFE was similar to that of the DDM-solubilized KcsA. Accordingly, the band corresponding to the dimer of tetramers had a $S_{20, w}$ value comparable to that observed for the solubilized protein. The area of this band was also similar under the two conditions studied (see, for comparison, panels B and F of Figure 6), with the observed minor differences being smaller than the experimental error. This suggests that the presence of lipid molecules in the solubilization medium does not modify significantly the tendency of the KcsA tetramer to selfassociate into clusters. At $12 \%$ TFE, a reduction was observed in the area of the bands corresponding to such clusters of KcsA, which were again no longer detectable at $18 \%$ TFE (Figure 6H). Taken together, the anisotropy and analytical ultracentrifugation results indicate that the supratetrameric assemblies of KcsA are disassembled at low TFE concentrations and that the TFE resistance of these higher-order oligomers is slightly higher when KcsA is solubilized in plain detergent micelles than in mixed micelles.

Reversibility of Cluster Assembly. The sedimentation equilibrium technique could not be satisfactorily employed to assess the reversibility of the KcsA cluster disassembly, as it requires high protein concentrations to yield accurate results. Therefore, in spite of its limitations, we decided to use SDS-PAGE, as it has been reported that electrophoretic bands of molecular mass higher than the tetramer can also be observed $(7,12)$. Accordingly, different KcsA samples were loaded in a $10 \%$ polyacrylamide gel, specially suited to study heavier protein species. It was observed that, in addition to the band corresponding to the tetramer, with an apparent molecular mass of $60 \mathrm{kDa}$, a less intense band of approximately $120 \mathrm{kDa}$ was present in the DDM-solubilized KcsA, likely corresponding to a "dimer" of KcsA tetramers (Figure 7, lane 1). We also observed a diffuse band with a mass slightly higher than that of the tetramer. This is reminiscent of the observation in KcsA electrospray ionization mass spectrometry experiments (ESI-MS) (29) of a peak with a mass slightly higher than that of the KcsA tetramer. It is conceivable that this electrophoretic band could perhaps arise from KcsA tetramers forming a stable complex with other bacterial components (30).

As expected, none of the above bands were observed in the gel at TFE concentrations leading to the dissociation into subunits of the KcsA tetramer [37\% TFE (v/v)] (lane 2). Interestingly, dilution of the TFE concentration in the previous sample below 5\% TFE (by dilution and partial vacuum drying), leads not only to the reappearance of the tetrameric KcsA band but also to an important recovery of the $120 \mathrm{kDa}$ band (lane 3). Unfortunately, the extent of the recovery cannot be quantified accurately, since after the drying step a substantial pellet was observed in the samples that could not be solubilized by the SDS-containing sample 
$0 \%$ TFE
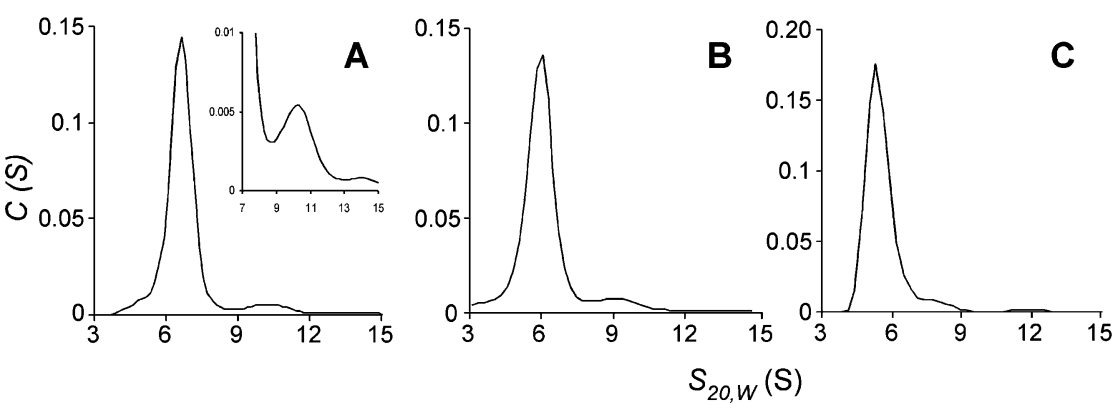

$6 \%$ TFE
$12 \%$ TFE

$18 \%$ TFE
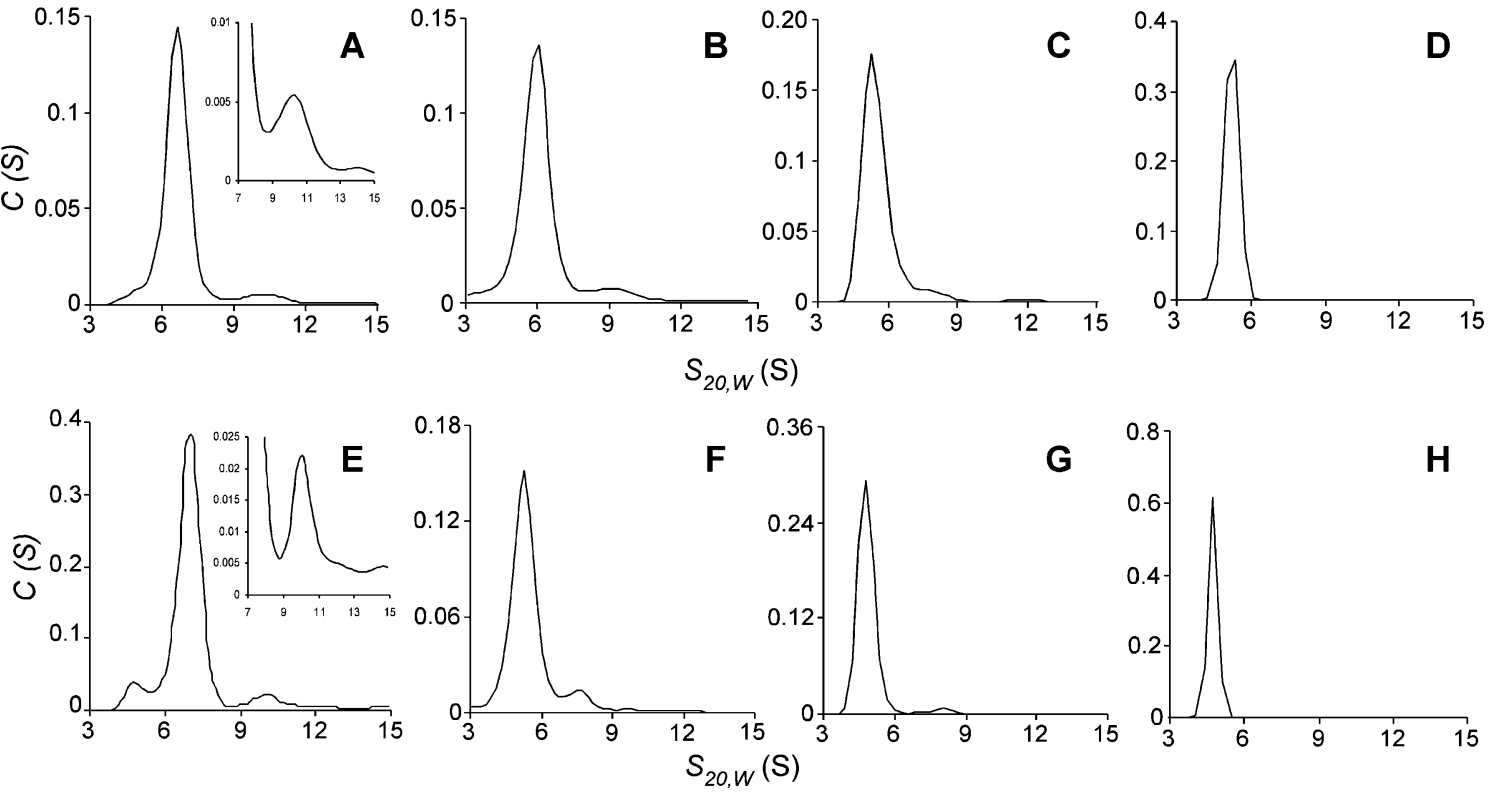

FIGURE 6: Differential sedimentation coefficient distributions of KcsA in the presence of TFE. (Upper row) KcsA solubilized in DDM, in the presence of (A) $0 \%$, (B) $6 \%$, (C) $12 \%$, and (D) $18 \%$ TFE (v/v). (Lower row) KcsA solubilized in mixed micelles of DDM and DOPE/ DOPG (7:3) in the presence of (E) $0 \%$, (F) 6\%, (G) 12\%, and (H) 18\% TFE (v/v). The indicated $S_{20, w}$ values were obtained after viscosity and density corrections (see Materials and Methods). The insets in panels A and E zoom on the higher sedimentation coefficient components of these samples. The DDM concentration was $5 \mathrm{mM}$, and in the mixed micelles samples, a lipid to protein ratio of 23:1 was used. The confidence level ( $F$-ratio) employed in the sedimentation coefficient distribution analysis was higher than that used previously ( 7$)$, in order to increase the reliability of the results. This made bands accounting for a low percentage of the total signal appear flattened. The temperature was $20^{\circ} \mathrm{C}$.

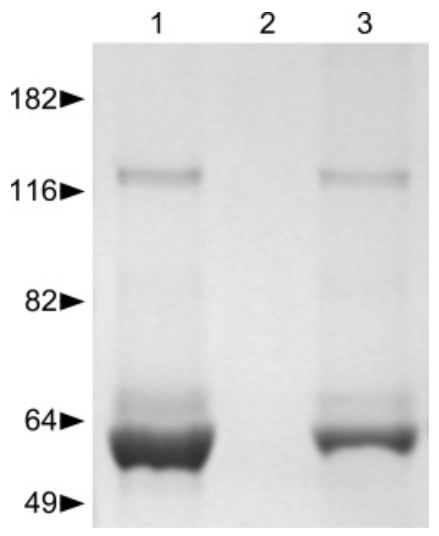

FIGURE 7: SDS-PAGE analysis of KcsA cluster reassembly. Lane 1: Control DDM-solubilized KcsA $(23 \mu \mathrm{M})$. The more intense band corresponds to the KcsA tetramer. Lane 2: DDM-solubilized KcsA in the presence of $37 \%$ TFE (v/v), which causes all bands to disappear. Lane 3: KcsA samples first treated as in lane 2 and then diluted 2-fold in DDM buffer, partially evaporated under vacuum, and diluted with water to reduce the TFE final concentration below $5 \%(\mathrm{v} / \mathrm{v})$. Polyacrylamide gels $(10 \%)$ were used in these experiments. The positions of protein size markers (in kilodaltons) are shown on the left.

buffer (see Materials and Methods). Sample precipitation also precluded other detailed quantitative studies, such as analysis of the effect of lipids in the reassembly of the dimers of KcsA channels. Nevertheless, in spite of the lack of quantitative sample recovery, the results show that the refolded KcsA obtained after removal of the TFE is still able to assemble into supramolecular, SDS-resistant dimers of channels, which are considered the building blocks of the larger KcsA clusters. In fact, comparison of the relative intensities of the bands at 60 and $120 \mathrm{kDa}$ in lanes 1 and 3 shows that their ratio in the refolded KcsA samples is comparable to that observed in the native protein, suggesting that the disassembly of the KcsA clusters is indeed a reversible process.

\section{DISCUSSION}

The preliminary exploration by SDS-PAGE of the reversibility of the KcsA unfolding by TFE revealed that the presence of lipids increased the efficiency of tetramer refolding upon TFE dilution (Figure 1). In the presence of DOPE/DOPG (7:3) in particular, the intensity of the electrophoretic band corresponding to the tetramer was very similar to that of the native protein, suggesting that a complete refolding had been attained under these conditions. This observation seems reminiscent of that of van Dalen et al. (23) with an in vitro membrane insertion and assembly assay, which showed that the highest level of KcsA tetramer insertion into liposomes is obtained with the mixture DOPE/ DOPG (7:3). Such mixture somewhat resembles the phospholipid composition of the inner membrane of the strains Streptomyces ambofaciens (31) and Streptomyces hygroscopicus (32), where the main components are PE $(\sim 50 \%)$, and negatively charged phospholipids $(\sim 40 \%)$ (the lipid composition of $S$. lividans appears not to have been determined).

Here we have carried out the characterization of the unfolding and refolding of KcsA in mixed micelles of DDM and DOPE/DOPG (7:3). We favored this alternative over the classical reconstitution of the protein into lipid bilayers, as the use of mixed micelles has several experimental advantages: (i) the samples are transparent, which facilitates the use of spectroscopical methods, while the reconstituted samples are somewhat cloudy and scattering artifacts are 
sometimes difficult to avoid; (ii) there are no turbidity changes associated with the addition of TFE, while the solubilization of liposomes by TFE is accompanied by a marked reduction in sample turbidity (10), in a process somewhat comparable to the complex detergent-induced phase change from bilayer to detergent-solubilized phospholipids $(33,34)$; and (iii) in mixed micelles, as the protein is not inserted into a lipid bilayer, the interpretation of the results is more straightforward, as the results of the effects of lipids are not influenced by other lipid-related changes such as the physical properties of the membrane bilayer, such as the lateral pressure $(28,35)$.

In the presence of increasing TFE concentrations, we observed that the tetrameric KcsA solubilized in mixed micelles of detergent and DOPE/DOPG unfolded in a sigmoidal fashion (Figures 3 and 5B). This is similar to that previously reported for KcsA solubilized in plain detergent micelles (11). However, in contrast to that previous report, the refolding in the mixed micelle system was seemingly quantitative (Figures 3 and 4B). This allowed the fitting of the tetramer to monomer transition to a two-state equation describing the unfolding/refolding equilibrium process, which yielded a value for the $\Delta G$ of the KcsA tetramer of $30.5 \pm$ $3.1 \mathrm{kcal} \cdot \mathrm{mol}^{-1}$. Such a value cannot be compared with that of other tetrameric helical membrane proteins because, to the best of our knowledge, KcsA is the first such protein for which the $\Delta G$ value has been determined. However, the stability of KcsA can be compared with those of soluble tetrameric proteins that also unfold through a two-state mechanism. For instance, the stability of proteins of a similar size to that of KcsA, such as transthyretin (36) and the chaperone SecB (37) (with monomers of 127 and 154 amino acids, respectively), is only slightly lower (29.1 and 27.9 $\mathrm{kcal} \cdot \mathrm{mol}^{-1}$, respectively) than that of KcsA. In the case of the p53 tetramerization domain, with monomers of 56 amino acids, the free energy of unfolding is $32.5 \mathrm{kcal} \cdot \mathrm{mol}^{-1}$ (27). Then, the determined stability of KcsA seems similar to that of tetrameric water-soluble proteins of similar size. This is not unprecedented, as similar conclusions were extracted in the case of the monomeric membrane protein OmpA, a $\beta$-barrel porin (38).

The obtained refolding results must be interpreted bearing in mind that when KcsA is purified in DDM (see Materials and Methods), the protein is not lipid-depleted. In fact, a lipid molecule tentatively identified as PG is observed in the crystal structure of KcsA at each monomer-monomer interface, in the vicinity of $\operatorname{Trp} 87(9,39)$. ESI-MS experiments also show that the interaction of KcsA with PG molecules is very strong, while the interaction with PE and PC is less stable (29). Phosphate quantification analysis of KcsA samples purified by a protocol very similar to that employed here, established that KcsA contains approximately $0.7 \mathrm{~mol}$ of phospholipid/mol of KcsA monomer, indicating that, on the average, $\sim 1$ out of 4 of the lipid-binding sites in KcsA is empty (9). As we have observed that the presence of lipids increases the refolding efficiency of KcsA, it is tempting to hypothesize that the high refolding level (80\%) observed for the DDM-solubilized KcsA (i.e., in the absence of added lipids), may be due to, or at least favored by, the lipid that copurifies with KcsA. Thus, lipid molecules present in the mixed micelles would bind to the empty lipid binding sites, enabling more KcsA monomers to refold into tetramers.
From this viewpoint, the lipid could be considered as an effector in the tetramerization of KcsA, similarly to that described for the light harvesting complex of photosystem II, where PG is required for the formation of functional trimers (40). In order to test this hypothesis, we submitted KcsA samples to extensive dialysis in buffer containing $\mathrm{DDM}$, in an attempt to remove the KcsA-bound lipid. Unfortunately, the lipids could not be removed by this procedure, as assessed by TLC of the dialyzed samples (data not shown), and thus the direct effect of the native KcsAbound lipid in the refolding of KcsA could not be addressed.

Monitoring the effects of TFE on KcsA by fluorescence anisotropy allowed the detection of an additional cooperative transition at low TFE concentrations. This transition went unnoticed when other spectroscopic parameters, such as fluorescence intensity or circular dichroism (11), were used in the monitoring of the process, and thus it is to be expected that it relates to the conversion between KcsA states with very similar secondary structure and solvent exposure but subjected to different dynamics. Furthermore, analytical ultracentrifugation results for KcsA solubilized in DDM and in mixed micelles showed that the protein sedimented predominantly as a tetramer, although a small populations of higher order clusters (mostly ensembles of two tetrameric KcsA molecules) were also detected, accounting for $\sim 10 \%$ of the total KcsA population (Figure 6 and ref 7). These supratetrameric assemblies were disrupted within the low TFE concentration range causing the anisotropy transition from above, and therefore, we attributed such transition to the disassembly of the small population of clusters into individual KcsA tetramers. This process occurs similarly in KcsA solubilized in plain DDM micelles or in mixed micelles, as the percentage of the tetrameric KcsA able to dimerize into clusters in DOPE/DOPG mixed micelles was the same, within the error, as that in the DDM-solubilized protein. This is in stark contrast to the high levels of protein self-assembly observed in KcsA reconstituted in giant liposomes, resulting in large (up to micrometer-size) clusters (7). Taken together, these observations suggest that the role of the lipid molecules as effectors is restricted to the formation of KcsA tetramers from unfolded protein subunits, while the formation of larger clusters of channels seems to require that lipids are present in the form of a well-defined bilayer. In regard to the possible protein-lipid interactions involved, it could be of interest to note that at a TFE concentration of $18 \%(\mathrm{v} / \mathrm{v})$, which causes the complete disassembly of the clusters into individual KcsA tetramers, the $S_{20, \mathrm{w}}$ values of the tetramer in mixed micelles was $4.8 \mathrm{~S}$, while that in the DDM-solubilized protein was 5.4 S. This difference can be explained by the tight binding of lipid molecules to KcsA, assuming that the association of the lipid does not cause important changes in the overall shape of the protein (41). This is due to the fact that the partial specific volume of the phospholipids $\left[0.98 \mathrm{~cm}^{3} / \mathrm{g}\right.$ for DOPE and 0.91 $\mathrm{cm}^{3} / \mathrm{g}$ for DOPG (42)] is higher than that of KcsA $(0.74$ $\mathrm{cm}^{3} / \mathrm{g}$ ). Hence, the KcsA-bound lipid molecules have a buoyancy effect and reduce the density of the complex.

The $[\mathrm{TFE}]_{1 / 2}$ of the transitions associated with the dissociation of both the dimer of tetramers and the individual KcsA tetramers seemed to be lower in mixed micelles than in detergent micelles. This occurred at all the different KcsA concentrations employed (data not shown) and could be 
related to previous results showing that the secondary structure of KcsA is slightly different in its solubilized and lipid-reconstituted forms (43). This suggests that the binding of lipid molecules could induce a conformational change in KcsA, leading to a reduction of its resistance to TFE. Alternatively, it could be argued that the small observed differences in tetramer stability could be due to changes in the TFE concentration required to dissolve the different micelles. We do not favor this possibility, as we have observed that the TFE concentration required to completely dissolve micelles of DDM at $5 \mathrm{mM}$ [30\% TFE (v/v) (11)] was identical to that needed to dissolve mixed micelles of DDM supplemented with DOPE/DOPG (data not shown).

The SDS-PAGE results on the reversibility of disassembly of the DDM-solubilized KcsA clusters (Figure 7) indicate that, upon TFE dilution, the unfolded KcsA monomers are able to fold into native tetramers, which still retain the ability to self-associate into higher order oligomers. Therefore, within the limitations of the SDS-PAGE technique used in these studies, the disassembly of KcsA clusters (dimers of tetramers) into individual KcsA tetramers should be considered as a reversible process.

The above dimers of tetramers of KcsA channels can be considered the building blocks of the larger KcsA clusters (7) and the detection of their reversible dissasembly complements previous unfolding/refolding results (11), allowing a more detailed description of the steady-state folding mechanism of KcsA, which can be summarized in the following scheme:

$$
\mathrm{T}_{n} \rightleftarrows \mathrm{T} \rightleftarrows \mathrm{M} \rightarrow \mathrm{D}
$$

where clusters of KcsA channels $\left(T_{n}\right)$, mainly in the form of dimers of tetramers under our experimental conditions (i.e., $n \sim 2$ ), are reversibly disassembled in the presence of low TFE concentrations into individual KcsA tetramers (T). Further increasing the TFE concentration up to moderate levels makes these tetramers break apart into unfolded monomers $(\mathrm{M})$, in a process that is completely reversible in the presence of low concentrations of DOPE and DOPG, which suggests a role of specific lipids as effectors in the protein oligomerization process. Finally, high TFE concentrations cause these monomers to denaturate irreversibly into a highly helical state (D) (11). An obvious corollary to these observations is that a functional oligomeric, helical membrane protein can fold and assemble in vitro without the assistance of other proteins such as the translocon complex or chaperones.

\section{ACKNOWLEDGMENT}

We thank Drs. Germán Rivas and Carlos Alfonso (Centro de Investigaciones Biológicas, CSIC, Madrid) for help with the analytical ultracentrifugation experiments and Dr. Daniel Otzen (Department of Life Sciences, Aalborg University, Denmark) for help with the anisotropy measurements. We also thank Dr. José Luis Neira from our Institute for his comments on the manuscript and Mrs. Eva Martínez for excellent technical help.

\section{SUPPORTING INFORMATION AVAILABLE}

Fitting to eq 3 of the data from Figure 3A, performed with different values for the $\beta_{\mathrm{D}}$ parameter, set to different fixed values, resulting in an upper plateau with a slope either positive or negative, or let free to fluctuate, showing that the obtained $\Delta G$ values were similar in all cases, indicating that the free energy value determined in the paper is not importantly influenced by the slope of the "unfolded" plateau. This material is available free of charge via the Internet at http://pubs.acs.org.

\section{REFERENCES}

1. Hessa, T., Kim, H., Bihlmaier, K., Lundin, C., Boekel, J., Andersson, H., Nilsson, I., White, S. H., and von Heijne, G. (2005) Recognition of transmembrane helices by the endoplasmic reticulum translocon, Nature 433, 377-381.

2. Booth, P. J., and Curnow, P. (2006) Membrane proteins shape up: understanding in vitro folding, Curr. Opin. Struct. Biol. 16, $480-488$

3. Lau, F. W., and Bowie, J. U. (1997) A method for assessing the stability of a membrane protein, Biochemistry 36, 5884-5892.

4. Otzen, D. E. (2003) Folding of DsbB in mixed micelles: a kinetic analysis of the stability of a bacterial membrane protein, $J$. Mol. Biol. 330, 641-649.

5. Schrempf, H., Schmidt, O., Kummerlen, R., Hinnah, S., Muller, D., Betzler, M., Steinkamp, T., and Wagner, R. (1995) A prokaryotic potassium ion channel with two predicted transmembrane segments from Streptomyces lividans, EMBO J. 14, 51705178.

6. Doyle, D. A., Morais, C. J., Pfuetzner, R. A., Kuo, A., Gulbis, J. M., Cohen, S. L., Chait, B. T., and MacKinnon, R. (1998) The structure of the potassium channel: molecular basis of $\mathrm{K}^{+}$ conduction and selectivity, Science 280, 69-77.

7. Molina, M. L., Barrera, F. N., Fernandez, A. M., Poveda, J. A., Renart, M. L., Encinar, J. A., Riquelme, G., and Gonzalez-Ros, J. M. (2006) Clustering and coupled gating modulate the activity in KcsA, a potassium channel model, J. Biol. Chem. 281, 1883718848.

8. Hegermann, J., Overbeck, J., and Schrempf, H. (2006) In vivo monitoring of the potassium channel KcsA in Streptomyces lividans hyphae using immuno-electron microscopy and energyfiltering, Microbiology 152, 2831-2841

9. Valiyaveetil, F. I., Zhou, Y., and MacKinnon, R. (2002) Lipids in the structure, folding, and function of the $\mathrm{KcsA} \mathrm{K}{ }^{+}$channel, Biochemistry 41, 10771-10777.

10. van den Brink-van der Laan, E., Chupin, V., Killian, J. A., and de Kruijff, B. (2004) Stability of KcsA tetramer depends on membrane lateral pressure, Biochemistry 43, 4240-4250.

11. Barrera, F. N., Renart, M. L., Molina, M. L., Poveda, J. A., Encinar, J. A., Fernandez, A. M., Neira, J. L., and Gonzalez-Ros, J. M. (2005) Unfolding and refolding in vitro of a tetrameric, alpha-helical membrane protein: the prokaryotic potassium channel KcsA, Biochemistry 44, 14344-14352.

12. Molina, M. L., Encinar, J. A., Barrera, F. N., Fernandez-Ballester, G., Riquelme, G., and Gonzalez-Ros, J. M. (2004) Influence of $\mathrm{C}$-terminal protein domains and protein-lipid interactions on tetramerization and stability of the potassium channel KcsA, Biochemistry 43, 14924-14931.

13. Pace, C. N., and Scholtz, M. (1997) in Protein Structure: A Practical Approach (Creighton, T. E., Ed.) IRL Press at Oxford University Press, Oxford, U.K.

14. Laemmli, U. K. (1970) Cleavage of structural proteins during the assembly of the head of bacteriophage T4, Nature 227, 680685 .

15. Ladokhin, A. S., Jayasinghe, S., and White, S. H. (2000) How to measure and analyze tryptophan fluorescence in membranes properly, and why bother?, Anal. Biochem. 285, 235-245.

16. Ehrlich, L. S., Fong, S., Scarlata, S., Zybarth, G., and Carter, C. (1996) Partitioning of HIV-1 Gag and Gag-related proteins to membranes, Biochemistry 35, 3933-3943.

17. Lakowicz, J. R. (1999) in Principles of fluorescence spectroscopy, pp 291-318, Kluwer Academic/Plenum Publishers, New York

18. Backmann, J., Schafer, G., Wyns, L., and Bonisch, H. (1998) Thermodynamics and kinetics of unfolding of the thermostable trimeric adenylate kinase from the archaeon Sulfolobus acidocaldarius, J. Mol. Biol. 284, 817-833.

19. Mallam, A. L., and Jackson, S. E. (2006) Probing nature's knots: the folding pathway of a knotted homodimeric protein, J. Mol. Biol. 359, 1420-1436. 
20. Schuck, P. (2000) Size-distribution analysis of macromolecules by sedimentation velocity ultracentrifugation and Lamm equation modeling, Biophys. J. 78, 1606-1619.

21. Gonzalez, J. M., Velez, M., Jimenez, M., Alfonso, C., Schuck, P., Mingorance, J., Vicente, M., Minton, A. P., and Rivas, G. (2005) Cooperative behavior of Escherichia coli cell-division protein FtsZ assembly involves the preferential cyclization of long single-stranded fibrils, Proc. Natl. Acad. Sci. U.S.A 102, 18951900.

22. MacPhee, C. E., Perugini, M. A., Sawyer, W. H., and Howlett, G. J. (1997) Trifluoroethanol induces the self-association of specific amphipathic peptides, FEBS Lett. 146, 265-268.

23. van Dalen, A., Hegger, S., Killian, J. A., and de Kruijff, B. (2002) Influence of lipids on membrane assembly and stability of the potassium channel KcsA, FEBS Lett. 525, 33-38.

24. Castanho, M. A., Santos, N. C., and Loura, L. M. (1997) Separating the turbidity spectra of vesicles from the absorption spectra of membrane probes and other chromophores, Eur. Biophys. J. 26, 253-259.

25. Bhairi, S. M. (1997) A guide to the properties and uses of detergents in biology and biochemistry, Calbiochem-Novabiochem Corporation, San Diego, CA.

26. Knol, J., Sjollema, K., and Poolman, B. (1998) Detergent-mediated reconstitution of membrane proteins, Biochemistry 37, 1641016415.

27. Mateu, M. G., and Fersht, A. R. (1998) Nine hydrophobic side chains are key determinants of the thermodynamic stability and oligomerization status of tumour suppressor p53 tetramerization domain, EMBO J. 17, 2748-2758.

28. van den Brink-van der Laan, E., Chupin, V., Killian, J. A., and de Kruijff, B. (2004) Small alcohols destabilize the KcsA tetramer via their effect on the membrane lateral pressure, Biochemistry $43,5937-5942$.

29. Demmers, J. A., van Dalen, A., de Kruijff, B., Heck, A. J., and Killian, J. A. (2003) Interaction of the $\mathrm{K}^{+}$channel KcsA with membrane phospholipids as studied by ESI mass spectrometry, FEBS Lett. 541, 28-32.

30. Reusch, R. N. (1999) Streptomyces lividans potassium channel contains poly- $(R)-3$-hydroxybutyrate and inorganic polyphosphate, Biochemistry 38, 15666-15672.

31. Schauner, C., Dary, A., Lebrihi, A., Leblond, P., Decaris, B., and Germain, P. (1999) Modulation of lipid metabolism and spiramycin biosynthesis in Streptomyces ambofaciens unstable mutants, Appl. Environ. Microbiol. 65, 2730-2737.
32. Hoischen, C., Gura, K., Luge, C., and Gumpert, J. (1997) Lipid and fatty acid composition of cytoplasmic membranes from Streptomyces hygroscopicus and its stable protoplast-type L form, J. Bacteriol. 179, 3430-3436.

33. le Maire, M., Champeil, P., and Moller, J. V. (2000) Interaction of membrane proteins and lipids with solubilizing detergents, Biochim. Biophys. Acta 1508, 86-111.

34. Lichtenberg, D., Opatowski, E., and Kozlov, M. M. (2000) Phase boundaries in mixtures of membrane-forming amphiphiles and micelle-forming amphiphiles, Biochim. Biophys. Acta 1508, 1-19.

35. Cantor, R. S. (1997) The lateral pressure profile in membranes: a physical mechanism of general anesthesia, Biochemistry 36, 2339-2344.

36. Niraula, T. N., Haraoka, K., Ando, Y., Li, H., Yamada, H., and Akasaka, K. (2002) Decreased thermodynamic stability as a crucial factor for familial amyloidotic polyneuropathy, J. Mol. Biol. 320, 333-342.

37. Panse, V. G., Swaminathan, C. P., Aloor, J. J., Surolia, A., and Varadarajan, R. (2000) Unfolding thermodynamics of the tetrameric chaperone, SecB, Biochemistry 39, 2362-2369.

38. Hong, H., and Tamm, L. K. (2004) Elastic coupling of integral membrane protein stability to lipid bilayer forces, Proc. Natl. Acad. Sci. U.S.A. 101, 4065-4070.

39. Zhou, Y., Morais-Cabral, J. H., Kaufman, A., and MacKinnon, R. (2001) Chemistry of ion coordination and hydration revealed by a $\mathrm{K}^{+}$channel-Fab complex at 2.0 A resolution, Nature 414 , $43-48$.

40. Nussberger, S., Dorr, K., Wang, D. N., and Kuhlbrandt, W. (1993) Lipid-protein interactions in crystals of plant light-harvesting complex, J. Mol. Biol. 234, 347-356.

41. Dufour, J. P., and Goffeau, A. (1980) Molecular and kinetic properties of the purified plasma membrane ATPase of the yeast Schizosaccharomyces pombe, Eur. J. Biochem. 105, 145-154.

42. Marsh, D. (1990) CRC Handbook of Lipid Bilayers, CRC Press, Boca Raton, FL.

43. Encinar, J. A., Molina, M. L., Poveda, J. A., Barrera, F. N., Renart, M. L., Fernandez, A. M., and Gonzalez-Ros, J. M. (2005) The influence of a membrane environment on the structure and stability of a prokaryotic potassium channel, KcsA, FEBS Lett. 579, $5199-5204$.

BI700778C 